\title{
Chlamydia testing in contraceptive clinics: who, where, how and why?
}

\begin{abstract}
The problem
"We have been appalled by the crisis in sexual health we have heard about and witnessed during our inquiry. We do not use the word 'crisis' lightly but in this case it is appropriate. This is a major public health issue and the problems identified in this report must be addressed immediately." 1

Chlamydia is the commonest sexually transmitted infection (STI) in the UK. Between 2001 and 2002 there was a $14 \%$ rise in the number of cases of chlamydia diagnosed at genitourinary medicine (GUM) clinics. The highest rates are seen in young people, especially women aged under 25 years. In 2002, 16-19-year-old females had the highest chlamydia rate, at about $1 \%$, of females seen at clinics. ${ }^{2}$ However, since the data given are collected from GUM clinics rather than on a population basis, the real extent of the problem is unknown, and research in the community suggests that about $5-10 \%$ of girls in this age group are infected ${ }^{3}$ (and therefore substantial numbers are generally undiagnosed).

In response to this, the second wave of the chlamydia screening programme has been announced. This is not a screening programme by invitation (unlike cytology or breast screening). It is an opportunistic testing programme that involves contraceptive clinics and general practice, as well as some other sites, in the geographical areas participating.
\end{abstract}

\section{Is it appropriate for chlamydia testing and} management to take place in contraceptive clinics?

There are obvious practical problems to be faced if this is to happen, but is it an appropriate thing to do in the first place?

Are we seeing the right people, and are we seeing enough of them?

Just under 1.4 million 'new episodes' (i.e. new attendances after at least 3 months' absence) were seen at GUM clinics in England, Wales and Northern Ireland in 2001. ${ }^{1}$ In the same year, there were about 2.6 million attendances at family planning clinics (FPCs) in England alone, made by about 1.2 million women and 91000 men. The peak age for FPC attendance was 16-19 years; an estimated $23 \%$ of women in this age group visited a clinic during the year. ${ }^{2}$ Therefore, contraceptive clinics are well placed to provide a testing service, not only to those who come and request it but also to a much more vulnerable group, namely those who have never heard of chlamydia but who are young and having unprotected sex.

Why extend this work outside GUM clinics?

Testing has been shown to be acceptable in contraceptive clinics $^{4}$ and, as the authors of this paper point out, FPC staff have skills in sexual behaviour counselling.

GUM clinics are experiencing severe workload problems. The Health Select Committee's Report on Sexual Health describes a median waiting time for GUM appointments of 12 days for men and 10 days for women, but waits of up to 8 weeks are recorded. One GUM service also reports 400 people a week being turned away through lack of capacity. In this situation it seems absurd for women (and men) to have to register at a FPC for their contraception, and re-register at a GUM clinic for their chlamydia test. If resources are limited, a one-stop shop approach must be more economical, as well as more convenient for clients.

\section{If it is appropriate, are contraceptive clinics able to do it?}

Testing is now easier on account of the following factors: 1. New technology. Many older family planning staff can remember the time when it was realised that nongonococcal urethritis in men (only diagnosed using laborious microscopy) was linked to pelvic inflammatory disease and cervicitis in women. This was followed by chlamydia culture as a research technique, then the easy but less sensitive enzyme immunoassay test, and now DNA tests that can even be taken by the clients themselves. We are promised dipstick testing in the future, ${ }^{5}$ which if successfully developed will remove the need for sophisticated laboratories. The technology is now easy; the difficult part is talking about sex and helping young people with painful decisions about their lives - surely this is our forte?

2. Expanded staff roles. Nurses who do cervical smears can take chlamydia tests with little extra training. The role of nurses has recently expanded greatly, leading to more job satisfaction for all clinical staff and the chance to reassess our function as holistic sexual health providers.

\section{If we do the testing, what do we do with the positive results?}

The management of any STI depends on diagnosis, treatment and partner notification (and, if appropriate, follow-up).

In some areas $^{6}$ there is no problem with women and their partners attending a GUM clinic if chlamydia is diagnosed in a contraceptive clinic. In other areas 7,8 there is a huge problem, and full management in FPCs can be a much better option. ${ }^{9}$ The numbers of men seen in FPCs in England have risen from 80000 in 1998-1999 to 93000 in 2002-2003; most will be attending for condoms but this is a chance to offer urine testing for chlamydia as well. This is not just an urban problem; teenagers living in rural areas may find it difficult to organise a hospital appointment confidentially.

It has always been a principle of STI work that these diseases 'hunt in packs' and that the discovery of one should lead to the search for others. However, there have always been exceptions, for example, antenatal clinics have traditionally tested for syphilis (and are now testing for HIV) but do not always offer chlamydia and gonorrhoea screening, HIV testing programmes have been set up as outreach projects, and the cervical screening programme can be seen as screening for the late result of an STI. Local knowledge is vital. In many areas of the UK gonorrhoea is rare; in those areas (such as London, the West Midlands and the North West) where it is more common, chlamydia testing needs to include gonorrhoea or there is a danger that those people who are gonorrhoea-positive but chlamydianegative will be given false reassurance.

\section{The challenges}

Does this mean that FPCs are expected to turn themselves into GUM clinics overnight? No - it means doing whatever we can do well and safely, which may vary from 'simple' 
awareness raising and referral, to testing, to complete management and partner notification. Some challenges we face are as follows:

1. Getting funding for the extra work involved. Commissioners need to recognise that sexual health is managed in many different settings, and arrange for funding to be available for all of these. Long-term support is needed, not short-term projects. The recommended rates of pay for family planning staff are poor compared to, say, general practice or NHS Direct and this makes recruitment difficult. 2. Getting used to new work. Change can be painful as well as exciting, and this is not the first time services have faced it. The change in the 1960 s to seeing unmarried people in FPCs was hugely stressful at the time, and (more recently) many staff have found the increased numbers of young people in our clinics challenging.

3. Data collection and public health assessment. These are hugely important issues, but if testing is to be accessible it must not be limited by the need for complex reports and computerisation. Microbiology labs can provide information on the number of tests taken, where they were taken and the number of positives, and the chlamydia screening programme is leading on more detailed data collection.

Whose responsibility is it to manage this epidemic?

A public health crisis like this one provides an opportunity for everyone to do what they can manage safely and effectively, for team work and co-operation between all branches of medicine, and for the focus to be on the client, not the service provider. Family planning, GUM, general practice, public health, schools, and indeed everyone can work together safely and effectively if the political will is present.
Statements on funding and competing interests

Funding. None identified.

Competing interests. None identified.

Lesley Bacon, MFFP, MRCGP

Consultant in Sexual and Reproductive Health, Lewisham Primary Care Trust, Department of Reproductive Health, St Giles, St Giles Road, London SE5 7RN, UK. E-mail: lesley.bacon@lewishampct.nhs.uk

References

1 House of Commons Select Committee Report on Sexual Health, 22 May 2003. http://www.publications.parliament.uk.

2 NHS Contraceptive Services, England 2001-2002. http://www. statistics.gov.uk/.

3 Pimenta JM, Catchpole M, Rogers PA, et al. Opportunistic screening for genital chlamydial infection. II: Prevalence among healthcare attenders, outcome, and evaluation of positive cases. Sex Transm Infect 2003; 79: 22-27.

4 Noone A, Spiers A, Allardice G, et al. Opportunistic screening for genital Chlamydia trachomatis infection and partner follow-up in family planning clinics in three Scottish cities. J Fam Plann Reprod Health Care 2004; 30(2): 84-85

5 http://www.admin.cam.ac.uk/news/dp/2003121801.

6 Siddiqui F, Kirkman RJ, Chandiok S. Re-audit of referral compliance of chlamydia positive women from a family planning clinic. J Fam Plann Reprod Health Care 2004; 30(2): 86-87.

7 Wilkinson C, Massil H, Evans J. An interface audit of chlamydia testing by community family planning clinics and referral to hospital genitourinary medicine clinics. Br J Fam Plann 2000; 26: 206-209.

8 Vanhegan G, Wedgwood A. Do young people attend genitourinary medicine clinics when referred by a community-based Brook Advisory Centre? Br J Fam Plann 1999; 25: 23-24.

9 Evans J, Baraitser P, Cross J, et al. Managing genital infection in community family planning clinics: an alternative approach to holistic sexual health service provision. Sex Transm Infect 2004; (in press).

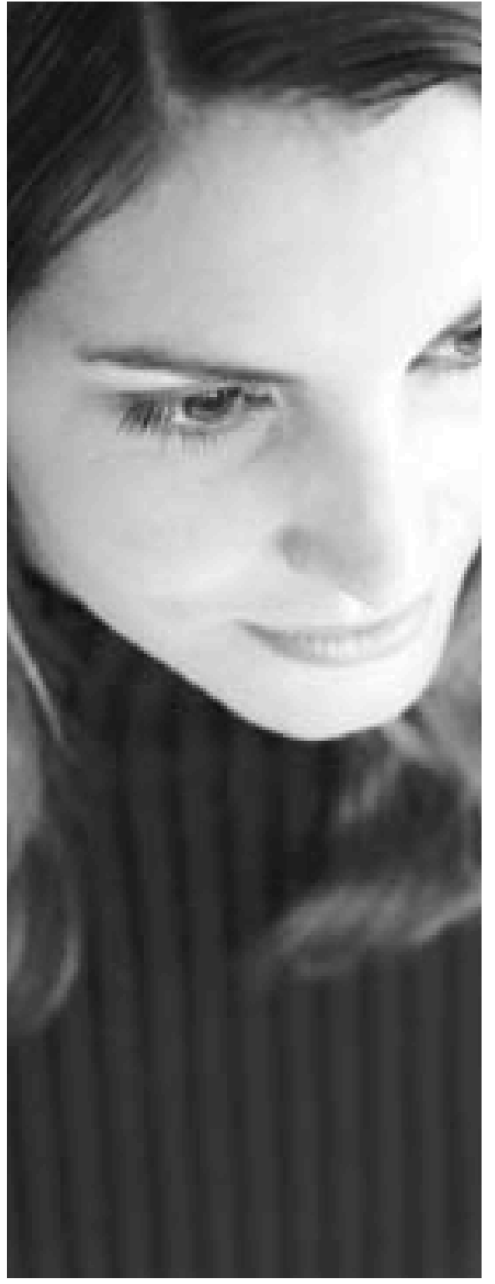

Termination of Pregnancy

\section{Fast, confidential, caring. The professional choice.}

Marie Stopes International is a leading charity in the field of family planning. We work closely with the NHS to provide termination of pregnancy services and are also happy to accept private referrals

At centres around the UK we offer a professional and convenient service. You can trust us to take care of the patients you refer. We're discreet, confidential, sympathetic and will do everything we can to make a difficult time easier. Patients can also rely on our full aftercare service including post abortion counselling.

If your patient would like an appointment or if you would like to find out more about us, simply call the number below. We will be happy to send you an information pack containing full details of our services and some easy to use referral forms. Alternatively, you can visit our web site

Call us on 08453001212 or visit www.mariestopes.org.uk

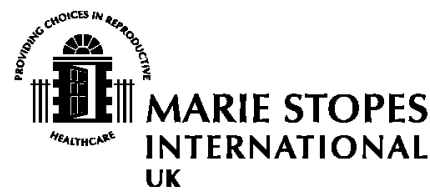

